\title{
Correlation between intraoperative ultrasound and postoperative MRI in pediatric tumor surgery
}

\author{
Heather Smith, BA, AmiLyn Taplin, MD, Sohail Syed, MD, and Matthew A. Adamo, MD \\ Department of Neurosurgery, Albany Medical Center, Albany, New York
}

OBJECTIVE Malignant disease of the CNS is the primary etiology for deaths resulting from cancer in the pediatric population. It has been well documented that outcomes of pediatric neurosurgery rely on the extent of tumor resection. Therefore, techniques that improve surgical results have significant clinical implications. Intraoperative ultrasound (IOUS) offers real-time surgical guidance and a more accurate means for detecting residual tumor that is inconspicuous to the naked eye. The objective of this study was to evaluate the correlation of extent of resection between IOUS and postoperative MRI. The authors measured the correlation of extent of resection, negative predictive value, and sensitivity of IOUS and compared them with those of MRI.

METHODS This study consisted of a retrospective review of the medical charts of all pediatric patients who underwent neurosurgical treatment of a tumor between August 2009 and July 2015 at Albany Medical Center. Included were patients who were aged $\leq 21$ years, who underwent brain or spinal tumor resection, for whom IOUS was used during the tumor resection, and for whom postoperative MRI (with and without contrast) was performed within 1 week of surgery.

RESULTS Sixty-two patients met inclusion criteria for the study ( 33 males, mean age 10.0 years). The IOUS results very significantly correlated with postoperative MRI results $(\varphi=0.726 ; p=0.000000011$; negative predictive value $86.3 \%$ $[95 \% \mathrm{Cl} 73.7 \%-94.3 \%])$. These results exemplify a $71 \%$ overall gross-total resection rate and $80 \%$ intended gross-total resection rate with the use of IOUS (i.e., excluding cases performed only for debulking purposes).

CONCLUSIONS The use of IOUS may play an important role in achieving a greater extent of resection by providing real-time information on tumor volume and location in the setting of brain shift throughout the course of an operation. The authors support the use of IOUS in pediatric CNS tumor surgery to improve clinical outcomes at low cost with minimal additional operating-room time and no identified additional risk.

http://thejns.org/doi/abs/10.3171/2016.5.PEDS15739

KEY WORDS intraoperative ultrasound; pediatric; tumor resection; neurosurgery; oncology

$\mathrm{M}$ ALIGNANT tumors of the CNS persist as the second most common overall pediatric malignancy, following only hematological disorders. CNS tumors are the primary cause of deaths resulting from cancer in children aged $0-14$ years. ${ }^{11,14}$ It is generally accepted that positive outcomes in pediatric neurosurgery depend significantly on the extent of tumor resection. ${ }^{12,13,15,16} \mathrm{In}$ light of these statistics, techniques that improve resection results have significant clinical implications in the pediatric population. In recent years, intraoperative ultrasound
(IOUS) has evolved into a widespread neuroimaging tool in neurosurgery that offers real-time surgical guidance. Using IOUS offers a more accurate means of detecting residual tumor not visible to the naked eye, thus rendering the best possible outcomes for pediatric patients.

Previous research established the intricate anatomical accuracy provided by IOUS. ${ }^{1}$ Tumors often appear hyperechoic in comparison with normal neural tissue as a result of the fact that echogenicity is physiologically related to cell density and extracellular components, ${ }^{6}$ both of which

ABBREVIATIONS IOUS = intraoperative ultrasound. 
are factors that change with malignancy and enable detection. Given its ability to differentiate subtle structural detail, IOUS has been used successfully to improve delineation of tumor margins and volumes. ${ }^{3,6,9,10,18,19}$ It can detect inhomogeneous echogenic areas of diffuse necrosis, hemorrhage, and cystic components of tumors, which are details often missed in traditional imaging studies. ${ }^{6}$ In comparison with CT and MRI, IOUS has proven to be more accurate in the differentiation between fluid-filled cystic and necrotic components of tumors. ${ }^{19}$ Most important is that this technique enables the surgeon to follow his or her progression through a tumor excision, providing information such as localization, margin delineation, and extent of tumor remaining in real-time dimensions of brain shift. ${ }^{18}$

As IOUS increasingly becomes incorporated into the neurosurgical armamentarium, its efficacy in determining the extent of tumor resection has been examined in populations of adults who have undergone neurosurgery. Unsgaard et al. ${ }^{18}$ compared the efficacy of using IOUS to using the naked eye. Once the surgeon had achieved grosstotal resection, one "last" IOUS scan revealed residual tumor in 53\% of the patients, consequently leading to further tumor removal and a greater extent of resection. Hammoud et al. ${ }^{7}$ compared the final IOUS scan to the postoperative MRI scan. In that study, of the 18 patients who had not had previous therapy, all of them had tumors with well-defined margins, and IOUS accurately determined the extent of resection. Chacko et al. ${ }^{2}$ performed a similar study that compared IOUS to postoperative MRI and also included histological samples from the brain-tumor interface to determine the sensitivity of IOUS. The tumor margins were well defined in $71.4 \%$ of the patients, all of whom had not had previous treatment. In tissue samples sent from sites at which IOUS revealed gross-total resection, 15 (88.2\%) of 17 indeed confirmed negative margins, thus suggesting an important new indication for IOUS. ${ }^{2}$

Furthermore, with improvements in ultrasonographic technology, IOUS may be used as an alternative to intraoperative MRI for determining the extent of tumor resection in real time. Using IOUS provides the advantage of lower cost, and IOUS is more readily available for general application in institutions that do not have intraoperative MRI capability. In addition, introducing the IOUS probe into the operative field in a sterile manner is simpler and less time-consuming than placing the operative field within the parameters of an MRI machine.

Up to now, only 2 existing studies have explored the use of IOUS in determining the extent of tumor resection in pediatric patients. ${ }^{5,17}$ The first study achieved an $82 \%$ overall gross-total resection rate and a $94 \%$ intended gross-total resection rate when excluding cases in which tumor was knowingly left behind. ${ }^{5}$ The second study included 22 cases in which complete resection was determined by the surgeon using IOUS, and only 1 case was later refuted by the neuroradiologist, who compared IOUS with the postoperative MRI. The authors emphasized the potential benefit of IOUS with tumor resection in younger patients and encouraged further investigation with more studies. ${ }^{17}$ Our study primarily aimed to evaluate the correlation of extent of resection between IOUS and postoper- ative MRI to provide further support for the role of IOUS in enhancing tumor surgery and negative margins. In this case series, we measured the correlation of extent of resection, negative predictive value, and sensitivity of IOUS and compared them with those of MRI.

\section{Methods}

This study consisted of a retrospective chart review of pediatric patients with CNS tumors treated surgically by a single pediatric neurosurgeon between August 2009 and September 2015 at Albany Medical Center in Albany, New York. All data were obtained from electronic medical records securely maintained in a central database in the hospital. Included were patients who were aged $\leq 21$ years, who underwent brain or spinal tumor resection, and who underwent IOUS during tumor resection and postoperative MRI with and without contrast within 1 week of surgery. Baseline characteristics were collected, as were clinical outcomes. The Albany Medical Center Committee on Research Involving Human Subjects approved this study.

First, a list of subjects was compiled from the pediatric neurosurgeon's billing list by identifying patients who had undergone resection of a brain or spinal cord tumor. For each patient on this list, the operative note, official pathology report, and official radiology report for the postoperative MRI were examined. Postoperative MRI with and without contrast was performed within 1 week of surgery, and the images were read by a board-certified radiologist. If postoperative changes obscured the final readout for residual tumor detection, the next postoperative MRI (performed within 3 months after surgery, per department policy) readout was examined. This second postoperative MRI was inspected in approximately half of the cases to improve accuracy of the results. The IOUS data were obtained from the operative report. This information was recorded, and the sensitivity and negative predictive values of IOUS were calculated by using the online version of MedCalc (www.medcalc.org). All other statistical analyses were performed with IBM SPSS Statistics for Windows, version 21.0.

\section{Technique}

All patients underwent preoperative neuronavigational MRI (Philips) with and without contrast. At the time of surgery, each patient was placed in a Mayfield pin head holder and secured to preferentially expose the intended surgical site. Brainlab was used for neuronavigation, and the preoperative images were registered to the patient. A craniotomy was performed, and once the dura mater was exposed, the surgical site was filled with a sterile saline solution to facilitate appropriate transduction of the acoustic beam between the IOUS probe and the dura. The sterile ultrasound probe (Hitachi Aloka Medical, Ltd., ProSound Alpha 5sx or Alpha7) was placed to define the tumor location and boundary. The relative location of the tumor was defined by scanning the surgical site for anatomical landmarks such as the ventricles or falx. Intraoperative ultrasound was conducted periodically throughout to assess the progression and appropriate trajectory of resection. 

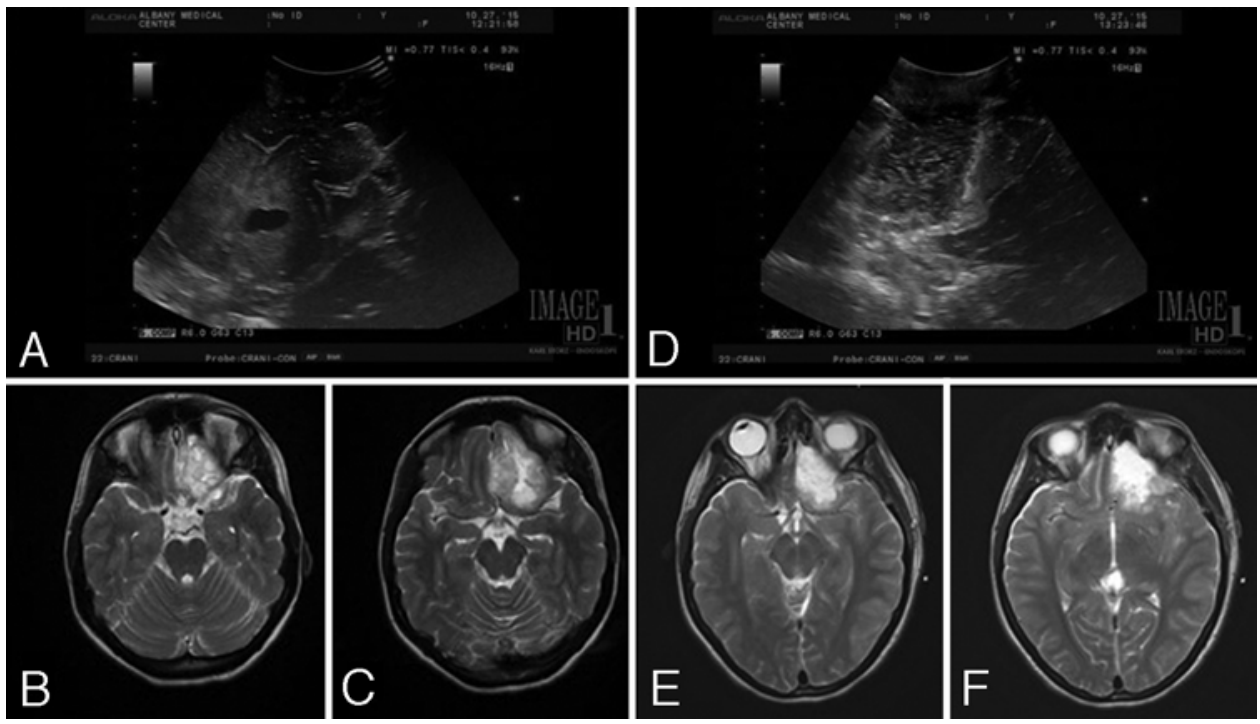

FIG. 1. Comparison of IOUS with preoperative and postoperative MRIs. A: Coronal ultrasound image showing a hyperechoic area in the left frontal lobe before resection. B and C: Axial T2-weighted MR images showing a mixed-intensity lesion in the left frontal lobe. D: Coronal ultrasound image showing a resection cavity that is hyperechoic along the periphery, indicating scattered blood products and edema. E and F: Axial T2-weighted MR images after resection revealing the resection cavity.

Once the tumor appeared completely resected by gross inspection, the ultrasound machine was brought back in for a final scan. If IOUS detected remnant tumor, resection was carefully continued until the final IOUS scan yielded a negative evaluation. Residual tumor in 11 patients was known at the time of surgery or even predicted during surgical planning because the tumor encroached on anatomically sensitive regions (such as the thalamus or brainstem). The use of IOUS became more valuable throughout the operation because of its ability to provide visualization in real time of relative tumor volume and location in the dynamic environment of resection.

In cases of spinal cord tumor, IOUS began once the laminectomy was performed and with the same technique as that for cases of cranial tumor but with a spine probe. The pediatric neurosurgeon manipulated the ultrasound probe within the surgical field and interpreted the images in real time. Two examples of IOUS imaging versus preoperative and postoperative MRI are provided in Figs. 1 and 2.
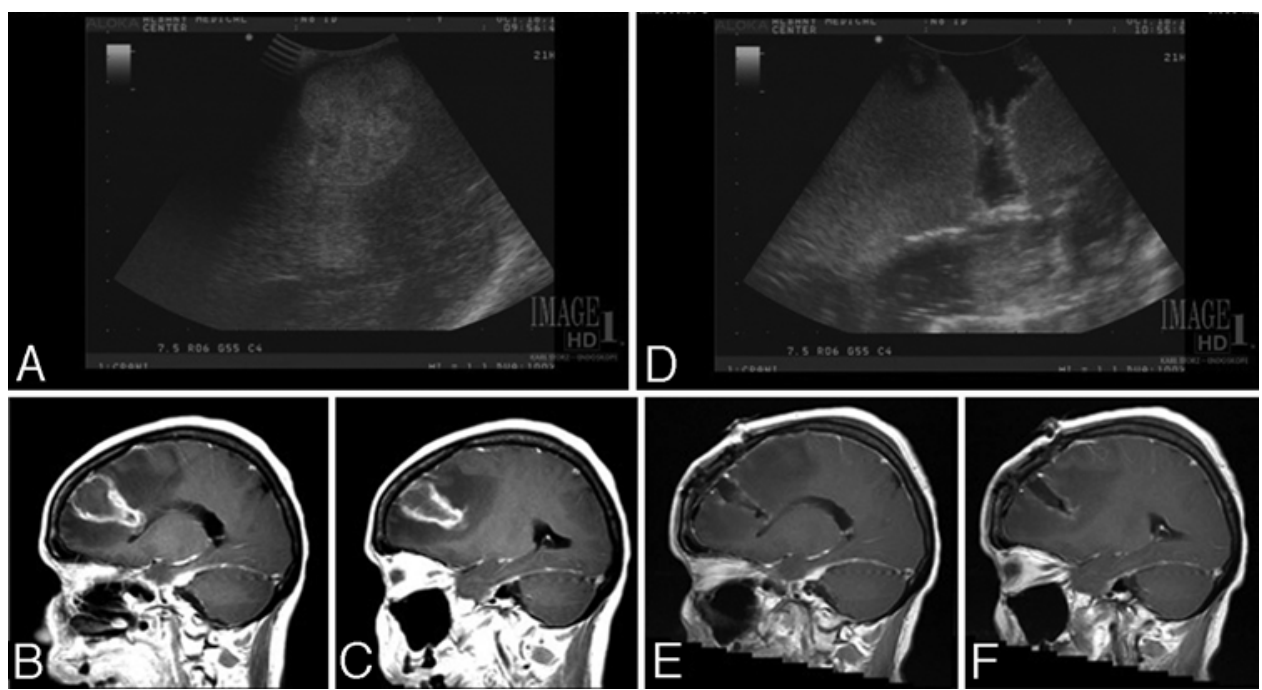

FIG. 2. Comparison of IOUS with preoperative and postoperative MRIs. A: Sagittal ultrasound image showing hyperechoic area in the left frontal lobe before resection; the left lateral ventricle is visible at the inferior edge of the lesion. B and C: Sagittal T1-weighted contrast-enhanced MR images showing a heterogeneously enhancing lesion in the left frontal lobe. D: Sagittal ultrasound image showing the resection cavity, which is hyperechoic along the periphery, indicating scattered blood products and blood in the lateral ventricle. E and F: Sagittal T1-weighted contrast-enhanced MR images after resection revealing the resection cavity. 
TABLE $1.2 \times 2$ table used for diagnostic test evaluation of IOUS

\begin{tabular}{lccc}
\hline \multirow{2}{*}{ IOUS } & \multicolumn{3}{c}{ Postoperative MRI } \\
\cline { 2 - 4 } & Residual Tumor & No Residual Tumor & Total \\
\hline Residual tumor & 11 & 0 & 11 \\
\hline No residual tumor & 7 & 44 & 51 \\
\hline Total & 18 & 44 & 62 \\
\hline
\end{tabular}

\section{Results}

A total of 62 patients met the inclusion criteria during the specified study period, including both newly diagnosed $(n=54)$ and recurrent $(n=8)$ tumors. Of the 62 patients, 33 were male and 29 were female. The mean age of the study patients was 10.0 years (range 3 months to 21 years).

The IOUS results correlated to a very significant degree with postoperative MRI results $(\varphi=0.726 ; \mathrm{p}=$ $0.000000011)$. The calculated negative predictive value of IOUS was $86.3 \%$ (95\% CI 73.7\%-94.3\%), and the sensitivity was $61.1 \%$ (95\% CI 35.8\%-82.7\%) (refer to Table 1 for a $2 \times 2$ diagnostic evaluation table). The IOUS and postoperative MRI results are further broken down according to tumor pathology in Table 2 .

Of 62 patients, $44(71 \%)$ demonstrated negative residual tumor on both IOUS and postoperative MRI. Eleven (18\%) patients showed residual tumor on both IOUS and postoperative MRI as a result of the tumor being located in an anatomically delicate area, whereas 7 patients (11\%) had a false-negative result (negative residual tumor on IOUS, positive residual tumor on MRI). These results exemplify a $71 \%$ overall gross-total resection rate and an $80 \%$ intended gross-total resection rate with the use of IOUS (i.e., excluding cases performed only for debulking purposes). Of the 7 false-negative tumors, $5(71 \%)$ were in the parietal lobe, whereas only 2 were in other areas of the brain (1 thalamic mass and 1 intraventricular mass). Overall, the study included 11 parietal lobe tumors, 5 (45\%) of which had a false-negative result. This is represented graphically in Fig. 3. The overall results are broken down further according to tumor location in Table 3. The false-negatives were distributed relatively evenly over the 7 years of the study, and no predominance in the beginning years of the surgeon's career was found. In addition, false-negative tumors varied from WHO Grade I to WHO Grade IV with no predilection for low- or high-grade tumors (shown in Table 4). Nineteen percent of the patients underwent additional surgeries for tumor resection, often because of recurrent tumor growth.

\section{Discussion}

CNS tumors remain the primary cause of death resulting from cancer in children aged 0-14 years, ${ }^{11,14}$ and maximal tumor resection has significant clinical implications for improving outcomes in the pediatric population. ${ }^{12,13,15,16}$ When determination for the extent of resection is being made, the effect on overall prognosis, degree of neurological morbidity from the tumor, and risk of neurological morbidity from surgery relative to the baseline neurological status must be taken into consideration. In
TABLE 2. Results according to tumor type according to official postoperative pathology reports

\begin{tabular}{|c|c|c|}
\hline Pathology & $\begin{array}{c}\text { No. of } \\
\text { Patients }\end{array}$ & IOUS/MRI (No. [\%]) \\
\hline Low-grade glioma & 34 & $\begin{array}{l}\text { Neg/Neg = } 24(71) \\
\text { Pos/Pos }=6(18) \\
\text { Neg/Pos }=4(12)\end{array}$ \\
\hline Pilocytic astrocytoma & 15 & $\begin{array}{l}\text { Neg/Neg }=11(73) \\
\text { Pos/Pos }=4(27) \\
\text { Neg/Pos }=0(0)\end{array}$ \\
\hline Low-grade astrocytoma & 5 & $\begin{array}{l}\text { Neg/Neg = } 2(40) \\
\text { Pos/Pos = } 1(20) \\
\text { Neg/Pos = } 2(40)\end{array}$ \\
\hline Low-grade glioma, unspecified & 4 & $\begin{array}{l}\text { Neg/Neg }=3(75) \\
\text { Pos/Pos }=1(25) \\
\text { Neg/Pos }=0(0)\end{array}$ \\
\hline $\begin{array}{l}\text { Myxopapillary ependymoma (1 } \\
\text { spinal intradural extramedullary) }\end{array}$ & 4 & $\begin{array}{l}\mathrm{Neg} / \mathrm{Neg}=4(100) \\
\mathrm{Pos} / \mathrm{Pos}=0(0) \\
\text { Neg/Pos }=0(0)\end{array}$ \\
\hline Fibrillary astrocytoma & 2 & $\begin{array}{l}\text { Neg/Neg }=2(100) \\
\text { Pos/Pos }=0(0) \\
\text { Neg/Pos }=0(0)\end{array}$ \\
\hline Pilomyxoid astrocytoma & 1 & $\begin{array}{l}\text { Neg/Neg }=0(0) \\
\text { Pos/Pos }=0(0) \\
\text { Neg/Pos }=1(100)\end{array}$ \\
\hline $\begin{array}{l}\text { Subependymal giant cell astro- } \\
\text { cytoma }\end{array}$ & 1 & $\begin{array}{l}\text { Neg/Neg }=0(0) \\
\text { Pos/Pos }=0(0) \\
\text { Neg/Pos }=1(100)\end{array}$ \\
\hline Oligodendroglioma & 1 & $\begin{array}{l}\text { Neg/Neg }=1(100) \\
\text { Pos/Pos }=0(0) \\
\text { Neg/Pos }=0(0)\end{array}$ \\
\hline Ganglioma & 1 & $\begin{array}{l}\text { Neg/Neg }=1(100) \\
\text { Pos/Pos }=0(0) \\
\text { Neg/Pos }=0(0)\end{array}$ \\
\hline Ependymoma, unclassified grade & 5 & $\begin{array}{l}\text { Neg/Neg = } 4(80) \\
\text { Pos/Pos = } 1(20) \\
\text { Neg/Pos = } 0(0)\end{array}$ \\
\hline $\begin{array}{l}\text { High-grade astrocytoma (1 spinal } \\
\text { tumor was Pos/Pos) }\end{array}$ & 5 & $\begin{array}{l}\text { Neg/Neg = } 3(60) \\
\text { Pos/Pos = } 1(20) \\
\text { Neg/Pos = } 1(20)\end{array}$ \\
\hline $\begin{array}{l}\text { High-grade ependymoma ( } 2 / 4 \text { were } \\
\text { anaplastic ependymomas) }\end{array}$ & 4 & $\begin{array}{l}\text { Neg/Neg }=2(50) \\
\text { Pos/Pos }=0(0) \\
\text { Neg/Pos }=2(50)\end{array}$ \\
\hline $\begin{array}{l}\text { High-grade neuroectodermal tumor } \\
\text { (1 pineoblastoma was Neg/Neg) }\end{array}$ & 3 & $\begin{array}{l}\text { Neg/Neg = } 2(67) \\
\text { Pos/Pos }=1(33) \\
\text { Neg/Pos }=0(0)\end{array}$ \\
\hline Metastatic tumor & 3 & $\begin{array}{l}\text { Neg/Neg }=3(100) \\
\text { Pos/Pos }=0(0) \\
\text { Neg/Pos }=0(0)\end{array}$ \\
\hline Medulloblastoma & 2 & $\begin{array}{l}\text { Neg/Neg }=2(100) \\
\text { Pos/Pos }=0(0) \\
\text { Neg/Pos }=0(0)\end{array}$ \\
\hline $\begin{array}{l}\text { Anaplastic pleomorphic xanthoastro- } \\
\text { cytoma w/ leptomeningeal spread }\end{array}$ & 2 & $\begin{array}{l}\text { Neg/Neg }=2(100) \\
\text { Pos/Pos }=0(0) \\
\text { Neg/Pos }=0(0)\end{array}$ \\
\hline
\end{tabular}

CONTINUED ON PAGE $582 »$ 
» CONTINUED FROM PAGE 581

TABLE 2. Results according to tumor type according to official postoperative pathology reports

\begin{tabular}{lcl}
\hline \multicolumn{1}{c}{ Pathology } & $\begin{array}{c}\text { No. of } \\
\text { Patients }\end{array}$ & IOUS/MRI (No. [\%]) \\
\hline $\begin{array}{l}\text { Infiltrating granular cell astrocytoma } \\
\text { (spinal) }\end{array}$ & 1 & $\begin{array}{l}\text { Neg/Neg }=0(0) \\
\text { Pos/Pos }=1(100) \\
\text { Neg/Pos }=0(0)\end{array}$ \\
\hline $\begin{array}{l}\text { Choroid plexus carcinoma, WHO } \\
\text { Grade III }\end{array}$ & 1 & $\begin{array}{l}\text { Neg/Neg }=1(100) \\
\text { Pos/Pos }=0(0)\end{array}$ \\
\hline Craniopharyngioma & & Neg/Pos $=0(0)$ \\
& 1 & Neg/Neg $=1(100)$ \\
& & $\begin{array}{l}\text { Pos/Pos }=1(100) \\
\text { Neg/Pos }=0(0)\end{array}$ \\
\hline Epidermoid cyst & 1 & Neg/Neg $=1(100)$ \\
& & Pos/Pos $=0(0)$ \\
& & Neg/Pos $=0(0)$ \\
\hline
\end{tabular}

Neg/Neg = tumor not present on IOUS or postoperative MRI; Neg/Pos = tumor not present on IOUS but present on postoperative MRI; Pos/Pos = tumor present on IOUS and postoperative MRI.

this consecutive case series, we examined the application of IOUS to optimize surgical outcomes by having realtime intraoperative guidance throughout the tumor resection. Measures of tumor resection with the use of IOUS correlated highly significantly with postoperative MRI results, indicating that IOUS is an effective adjunctive tool. In our case series, we found a respectable negative predictive value of $86.3 \%$ with the inclusion of multiple tumor pathologies located throughout the CNS. Across the diverse surgical cases considered in this study, a $71 \%$ overall gross-total resection rate was achieved. Excluding cases in which tumors were intended only to be debulked given their anatomical location, the study reflected an $80 \%$ intended gross-total resection rate with the use of IOUS. Using IOUS did not confer any identifiable complications and contributed minimal additional time to surgery.

The results of our study stand in congruence with those of previously reported investigations, and we contribute more generalizability across tumor types and locations. El Beltagy et al. ${ }^{5}$ recently described the role of IOUS in pediatric CNS tumor resection. Using a conventional 2D 6.5$\mathrm{MHz}$ probe, they concluded that the technique was useful for delineating the border between tumor and healthy brain tissue and for detecting remnant tumor that otherwise would have been missed. This study achieved an $82 \%$ overall gross-total resection rate and $94 \%$ intended gross-total resection rate in a smaller, less representative cohort of 22 pediatric patients. Ulrich et al. ${ }^{17}$ replicated this research and stated that IOUS enabled more effective differentiation between tumor and healthy brain tissue.

Periodic IOUS throughout tumor surgery can assist the surgeon in achieving better resection margins in individual cases. The technique provides immediate information about tumor volume and location as resection proceeds under conditions of CSF efflux and brain shift. We can continuously adjust our plan accordingly to the point of gross-total resection. However, we knowingly leave remnant tumor behind in specific cases, depending on the

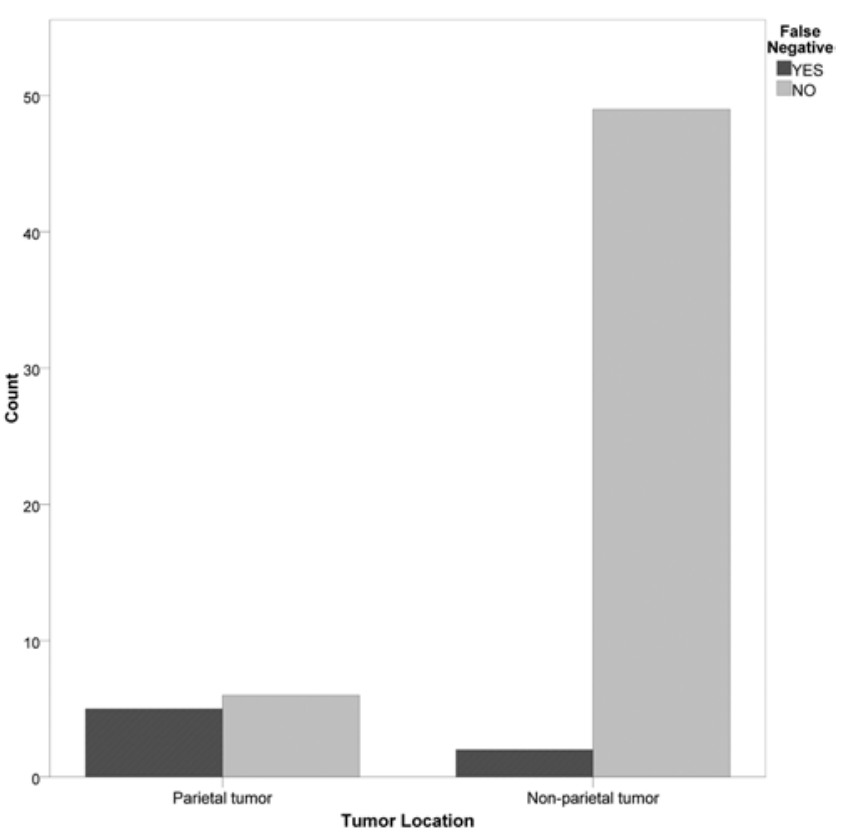

FIG. 3. False-negative rates based on tumor location: parietal versus nonparietal neoplasms. Five $(71 \%)$ of the 7 false-negatives were from a parietal mass ( $n=11$ parietal masses in the study overall).

gravity of the risk/benefit ratio. The ability to evaluate extent of resection and predict what the postoperative MRI will show is a powerful tool, just as meaningful as the ability to achieve negative margins in favorable cases. The highly significant correlation between IOUS and postoperative MRI lends confidence to the use of IOUS as a valuable tool in tumor resection.

Other neuronavigational modalities can guide tumor surgery, but intraoperative CT or MRI poses greater complexities. These intraoperative modalities significantly lengthen operating-room time, require additional staff to run the scanners, increase the risk of breaks in sterility, and have much higher overhead and running costs. Contrast is required for tissue demarcation, and ionizing radiation is emitted in the case of intraoperative CT. However, intraoperative MRI provides the advantage of eliminating the need for postoperative imaging and a second anesthetic-requiring scan. Intraoperative ultrasound offers a relatively simple means for surgical guidance with favorable risk/benefit and cost/benefit ratios.

Despite the high correlation of IOUS with the postoperative MRI in our case series, we do not propose that IOUS should supplant postoperative MRI. Postoperative MRI is necessary in all tumor cases, whether for a new baseline for surveillance imaging, radiation treatment planning, or both. We emphasize the utility of IOUS as an intraoperative tool that can provide information and guidance in real time. Using IOUS can certainly enhance the potential for greater rates of gross-total resection by providing intraoperative information, and the true benefit of IOUS is to show us how much tumor remains, if any, before making the decision to end the surgery based on the benefit/risk ratio.

In our practice, IOUS is used routinely in adult and pediatric patients with a CNS tumor, but we acknowledge 
TABLE 3. Results according to tumor location

\begin{tabular}{|c|c|c|}
\hline Location & No. of Patients & IOUS/MRI (No. [\%]) \\
\hline Intraventricular & 12 & $\begin{array}{l}\text { Neg/Neg }=8(66.7) \\
\text { Pos/Pos }=3(25) \\
\text { Neg/Pos }=1(8.3)\end{array}$ \\
\hline Cerebellum & 9 & $\begin{array}{l}\mathrm{Neg} / \mathrm{Neg}=9(100) \\
\mathrm{Pos} / \mathrm{Pos}=0(0) \\
\text { Neg/Pos }=0(0)\end{array}$ \\
\hline Occipital lobe & 6 & $\begin{array}{l}\text { Neg/Neg }=6(100) \\
\text { Pos/Pos }=0(0) \\
\text { Neg/Pos }=0(0)\end{array}$ \\
\hline Parietal lobe & 5 & $\begin{array}{l}\text { Neg/Neg }=3(60) \\
\text { Pos/Pos }=0(0) \\
\text { Neg/Pos }=2(40)\end{array}$ \\
\hline Frontal lobe & 5 & $\begin{array}{l}\mathrm{Neg} / \mathrm{Neg}=5(100) \\
\mathrm{Pos} / \mathrm{Pos}=0(0) \\
\text { Neg/Pos }=0(0)\end{array}$ \\
\hline Temporal lobe & 4 & $\begin{array}{l}\text { Neg/Neg }=3(75) \\
\text { Pos/Pos }=1(25) \\
\text { Neg/Pos }=0(0)\end{array}$ \\
\hline Parieto-occipital lobes & 4 & $\begin{array}{l}\text { Neg/Neg }=3(75) \\
\text { Pos/Pos }=0(0) \\
\text { Neg/Pos }=1(25)\end{array}$ \\
\hline Thalamus & 4 & $\begin{array}{l}\text { Neg/Neg }=3(75) \\
\text { Pos/Pos }=0(0) \\
\text { Neg/Pos }=1(25)\end{array}$ \\
\hline Frontoparietal lobes & 2 & $\begin{array}{l}\text { Neg/Neg }=0(0) \\
\text { Pos/Pos }=0(0) \\
\text { Neg/Pos }=2(100)\end{array}$ \\
\hline Midbrain & 2 & $\begin{array}{l}\text { Neg/Neg }=0(0) \\
\text { Pos/Pos }=2(100) \\
\text { Neg/Pos }=0(0)\end{array}$ \\
\hline Hypothalamus & 1 & $\begin{array}{l}\text { Neg/Neg }=0(0) \\
\text { Pos/Pos }=1(100) \\
\text { Neg/Pos }=0(0)\end{array}$ \\
\hline Pineal gland & 1 & $\begin{array}{l}\mathrm{Neg} / \mathrm{Neg}=0(0) \\
\mathrm{Pos} / \mathrm{Pos}=1(100) \\
\text { Neg/Pos }=0(0)\end{array}$ \\
\hline Pericallosal-periatrial & 1 & $\begin{array}{l}\text { Neg/Neg }=1(100) \\
\text { Pos/Pos }=0(0) \\
\text { Neg/Pos }=0(0)\end{array}$ \\
\hline Cervicomedullary spinal cord & 1 & $\begin{array}{l}\mathrm{Neg} / \mathrm{Neg}=0(0) \\
\mathrm{Pos} / \mathrm{Pos}=1(100) \\
\text { Neg/Pos }=0(0)\end{array}$ \\
\hline \multicolumn{3}{|l|}{ Spinal locations } \\
\hline Intradural extramedullary & 3 & $\begin{array}{l}\text { Neg/Neg }=3(100) \\
\text { Pos/Pos }=0(0) \\
\text { Neg/Pos }=0(0)\end{array}$ \\
\hline Intradural intramedullary & 2 & $\begin{array}{l}\text { Neg/Neg }=0(0) \\
\text { Pos/Pos }=2(100) \\
\text { Neg/Pos }=0(0)\end{array}$ \\
\hline
\end{tabular}

that IOUS has some limitations. A practical limitation is user dependency. As with many aspects of neurosurgery, user dependency is subject to a steep learning curve, but this limitation can be overcome readily with persistent use and instruction by an experienced neurosurgeon. Some institutions call a neuroradiologist into the operating room to interpret IOUS images. In this particular series, false-negative tumors were distributed fairly evenly over the 7 years and did not suggest user dependency to be a limiting factor in the study. IOUS requires a high-end ultrasound machine with a combined 5- to 7-MHz sector transducer and a 7- to $12-\mathrm{MHz}$ linear transducer for ideal neuroanatomical imaging. ${ }^{4}$ Although the image quality of ultrasound falls short of the exquisite detail of MRI, with a high-frequency linear transducer, one can differentiate the low-echogenic cortical ribbon from the brighter echogenicity of the subcortical white matter, and color Doppler sonography can easily evaluate tumor vascularity. ${ }^{4}$ Insonation depth can also be sequentially reduced on tumor examination to improve imaging with greater detail, ${ }^{4}$ and picture quality continues to improve with technological advances. Recent work with contrast-enhanced ultrasonography showed promising results for improving the visualization of tumors with ill-defined borders and differentiation between tumor and surrounding edematous brain tissue, which are 2 well-recognized limitations of IOUS. ${ }^{4}$

Another limitation of IOUS manifests in circumstances of recurrent tumors presenting for repeat resection or tumors that were irradiated previously. ${ }^{10}$ Gliosis affects the composition of brain matter and, thus, its acoustical propagation of sound. ${ }^{8}$ In a study by Hammoud et al., ${ }^{7}$ tumors were well localized in only $62 \%$ of the 13 patients who had undergone radiation therapy previously, and the extent of tumor resection was poorly defined. Similarly, Chacko et al. ${ }^{2}$ also reported ill-defined margins in patients with a previous history of radiation treatment.

Of the 7 patients in this study with a false-negative tumor, 2 had undergone previous surgery or radiation therapy for their CNS tumor. Another 3 of these 7 patients presented with a later-stage, more aggressive pathology. Last, 1 patient's tumor was diagnosed as tuberous sclerosis, which distorted the normal anatomy. Table 3 outlines the characteristics of these 7 subjects, including tumor type, location, and previous therapy. From this study, we cannot make significant comments on the correlation between IOUS and postoperative MRI based on tumor pathology or location. The diversity of pathology types limits the $\mathrm{N}$ value for any 1 classification, which makes any statistical analysis powerless. Qualitative observation reveals the parietal location to be more challenging in the evaluation of extent of tumor resection with IOUS, and there was a preponderance of false-negative results in this region.

\section{Conclusions}

The use of IOUS may play an important role in achieving a better extent of resection and improved clinical outcomes with minimal additional operating-room time and no identified additional risk and at low cost. The aid of IOUS enables us to make moment-to-moment decisions and adjustments to have a real impact on pediatric patients. Technological advances will improve image quality and enhance the role of IOUS in pediatric CNS tumor surgery, but we support the continued use of IOUS in the meantime given its favorable benefit/risk ratio. 
TABLE 4. Characteristics of false-negative cases

\begin{tabular}{ccllcll}
\hline $\begin{array}{c}\text { False-Negative } \\
\text { Patient No. }\end{array}$ & $\begin{array}{c}\text { Age } \\
\text { at Op } \\
\text { (yrs) }\end{array}$ & \multicolumn{1}{c}{ Tumor Histology } & $\begin{array}{c}\text { WHO } \\
\text { Grade }\end{array}$ & Location & $\begin{array}{c}\text { Previous } \\
\text { Op or } \\
\text { RT? }\end{array}$ & $\begin{array}{c}\text { Subsequent } \\
\text { Tumor } \\
\text { Resection? }\end{array}$ \\
\hline 1 & 3 & Large pilomyxoid astrocytoma & II & $\begin{array}{c}\text { Rt frontoparietal w/ exten- } \\
\text { sion to It frontal lobe }\end{array}$ & No & Yes \\
\hline 2 & 1 & Thalamopeduncular JPA & I/II & Lt thalamus \& midbrain & No & Yes \\
\hline 3 & 16 & Glioblastoma & IV & Parietooccipital & No & No \\
\hline 4 & 15 & $\begin{array}{c}\text { Subependymal giant-cell astrocy- } \\
\text { toma }\end{array}$ & I & Intraventricular & No & No \\
\hline 5 & 12 & Recurrent anaplastic ependymoma & III & Parietal & Yes & Yes \\
\hline 6 & 12 & Recurrent anaplastic ependymoma & III & Parietal & Yes & Yes \\
\hline 7 & 2 & Large infiltrating astrocytoma & II & Frontoparietal & No & No \\
\hline
\end{tabular}

JPA = juvenile pilocytic astrocytoma; PNET = primitive neuroectodermal tumor; $R T$ = radiotherapy.

\section{References}

1. Auer LM, van Velthoven V: Intraoperative ultrasound (US) imaging. Comparison of pathomorphological findings in US and CT. Acta Neurochir (Wien) 104:84-95, 1990

2. Chacko AG, Kumar NK, Chacko G, Athyal R, Rajshekhar V: Intraoperative ultrasound in determining the extent of resection of parenchymal brain tumours - a comparative study with computed tomography and histopathology. Acta Neurochir (Wien) 145:743-748, 2003

3. Chen SS, Shao KN, Chiang JH, Chang CY, Lao CB, Lirng $\mathrm{JF}$, et al: Intracranial pathology: comparison of intraoperative ultrasonography with computerized tomography and magnetic resonance imaging. Zhonghua Yi Xue Za Zhi (Taipei) 62:521-528, 1999

4. Cheon JE: Intraoperative neurosonography revisited: effective neuronavigation in pediatric neurosurgery. Ultrasonography 34:79-87, 2015

5. El Beltagy MA, Aggag M, Kamal M: Role of intraoperative ultrasound in resection of pediatric brain tumors. Childs Nerv Syst 26:1189-1193, 2010

6. Enzmann DR, Wheat R, Marshall WH, Bird R, MurphyIrwin K, Karbon K, et al: Tumors of the central nervous system studied by computed tomography and ultrasound. Radiology 154:393-399, 1985

7. Hammoud MA, Ligon BL, elSouki R, Shi WM, Schomer DF, Sawaya R: Use of intraoperative ultrasound for localizing tumors and determining the extent of resection: a comparative study with magnetic resonance imaging. J Neurosurg 84:737-741, 1996

8. Kremkau FW: Diagnostic Ultrasound: Physical Principles and Exercises. New York: Grune \& Stratton, 1980

9. Le Roux PD, Berger MS, Ojemann GA, Wang K, Mack LA: Correlation of intraoperative ultrasound tumor volumes and margins with preoperative computerized tomography scans. An intraoperative method to enhance tumor resection. J Neurosurg 71:691-698, 1989

10. Le Roux PD, Berger MS, Wang K, Mack LA, Ojemann GA: Low grade gliomas: comparison of intraoperative ultrasound characteristics with preoperative imaging studies. J Neurooncol 13:189-198, 1992

11. Linabery AM, Ross JA: Trends in childhood cancer incidence in the U.S. (1992-2004). Cancer 112:416-432, 2008

12. McGirt MJ, Chaichana KL, Attenello FJ, Weingart JD, Than $\mathrm{K}$, Burger PC, et al: Extent of surgical resection is independently associated with survival in patients with hemispheric infiltrating low-grade gliomas. Neurosurgery 63:700-708, 2008

13. McGirt MJ, Chaichana KL, Gathinji M, Attenello FJ, Than
$\mathrm{K}$, Olivi A, et al: Independent association of extent of resection with survival in patients with malignant brain astrocytoma. J Neurosurg 110:156-162, 2009

14. Ostrom QT, de Blank PM, Kruchko C, Petersen CM, Liao P, Finlay JL, et al: Alex's Lemonade Stand foundation infant and childhood primary brain and central nervous system tumors diagnosed in the United States in 2007-2011. Neuro Oncol 16 (Suppl 10):x1-x36, 2015

15. Safaee M, Oh MC, Mummaneni PV, Weinstein PR, Ames CP, Chou D, et al: Surgical outcomes in spinal cord ependymomas and the importance of extent of resection in children and young adults. J Neurosurg Pediatr 13:393-399, 2014

16. Sun MZ, Ivan ME, Clark AJ, Oh MC, Delance AR, Oh T, et al: Gross total resection improves overall survival in children with choroid plexus carcinoma. J Neurooncol 116:179-185, 2014

17. Ulrich NH, Burkhardt JK, Serra C, Bernays RL, Bozinov O: Resection of pediatric intracerebral tumors with the aid of intraoperative real-time 3-D ultrasound. Childs Nerv Syst 28:101-109, 2012

18. Unsgaard G, Ommedal S, Muller T, Gronningsaeter A, Nagelhus Hernes TA: Neuronavigation by intraoperative three-dimensional ultrasound: initial experience during brain tumor resection. Neurosurgery 50:804-812, 2002

19. van Velthoven V: Intraoperative ultrasound imaging: comparison of pathomorphological findings in US versus CT, MRI and intraoperative findings. Acta Neurochir Suppl 85:95-99, 2003

\section{Disclosures}

The authors report no conflict of interest concerning the materials or methods used in this study or the findings specified in this paper.

\section{Author Contributions}

Conception and design: all authors. Acquisition of data: Adamo. Analysis and interpretation of data: Adamo, Smith, Taplin. Drafting the article: Adamo, Smith, Taplin. Critically revising the article: Adamo, Smith, Taplin. Reviewed submitted version of manuscript: all authors. Approved the final version of the manuscript on behalf of all authors: Adamo. Statistical analysis: Smith. Administrative/technical/material support: Adamo, Smith, Syed. Study supervision: Adamo, Taplin, Syed.

\section{Correspondence}

Matthew A. Adamo, AMC Neurosurgery Group, 47 New Scotland Ave., MC 10, Albany, NY 12208. email: adamom@mail. amc.edu. 